\title{
Mitochondrial bioenergetics and D-ribose in HFpEF: a brief narrative review
}

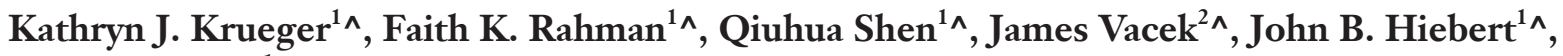 \\ Janet D. Pierce ${ }^{{ }^{\wedge}}$
}

${ }^{1}$ School of Nursing, University of Kansas Medical Center, Kansas City, KS, USA; ${ }^{2}$ The University of Kansas Health System, Kansas City, KS, USA Contributions: (I) Conception and design: All authors; (II) Administrative support: KJ Krueger, FK Rahman; (III) Provision of study materials or patients: None; (IV) Collection and assembly of data: None; (V) Data analysis and interpretation: None; (VI) Manuscript writing: All authors; (VII) Final approval of manuscript: All authors.

Correspondence to: Kathryn J. Krueger, MPH, CPH. School of Nursing, University of Kansas Medical Center, Mail Stop 4043,3901 Rainbow Blvd, Kansas City, KS 66160, USA. Email: kkrueger3@kumc.edu.

Objective: In this review article, we briefly describe the status of treatment options for HFpEF and the role of mitochondrial dysfunction in the pathogenesis of HFpEF as an alternative therapeutic target. We also examine the mechanisms of D-ribose in cellular energy production and discuss the potential disadvantages and benefits of supplemental use of D-ribose in patients with HFpEF.

Background: Heart failure is a major cardiovascular disease that impacts over 6 million Americans and is one of the leading causes for morbidity and mortality. Patients with heart failure often experience shortness of breath and fatigue along with impaired physical capacity, all leading to poor quality of life. As a subtype of heart failure, heart failure with preserved ejection fraction ( $\mathrm{HFpEF}$ ) is characterized with impaired diastolic function. Currently, there are no effective treatments specifically for HFpEF, thus clinicians and researchers are searching for therapies to improve cardiac function. Emerging evidence indicate that mitochondrial dysfunction and impaired cardiac bioenergetics are among the underlying mechanisms for HFpEF. There is increased interest in investigating the use of supplements such as D-ribose to enhance mitochondrial function and improve production of adenosine triphosphate (ATP).

Methods: For this narrative review, more than 100 relevant scientific articles were considered from various databases (e.g., PubMed, Web of Science, CINAHL, and Google Scholar) using the keywords "Heart Failure", "HFpEF”, "D-ribose”, “ATP”, "Mitochondria”, Bioenergetics”, and "Cellular Respiration”.

Conclusions: It is essential to find potential targeted therapeutic treatments for HFpEF. Since there is evidence that the $\mathrm{HFpEF}$ is related to impaired myocardial bioenergetics, enhancing mitochondrial function could augment cardiac function. Using a supplement such as D-ribose could improve mitochondrial function by increasing ATP and enhancing cardiac performance for patients with HFpEF. There is a recently completed clinical trial with HFpEF patients that indicates D-ribose increases ATP production and improves cardiac ejection fraction.

Keywords: Heart failure with preserved ejection fraction (HFpEF); D-ribose; mitochondria; adenosine triphosphate

Submitted May 04, 2021. Accepted for publication Aug 15, 2021.

doi: 10.21037/atm-21-2291

View this article at: https://dx.doi.org/10.21037/atm-21-2291

^ ORCID: Kathryn J. Krueger, 0000-0002-2997-8809; Faith K. Rahman, 0000-0002-9598-3972; Qiuhua Shen, 0000-0002-1236-0244; James Vacek, 0000-0001-8241-6381; John B. Hiebert, 0000-0001-8841-0378; Janet D. Pierce, 0000-0001-7100-7865. 


\section{Introduction}

\section{Background}

Heart disease is a common disorder that continues to rank as the leading cause of mortality in the United States (U.S.) and worldwide, accounting for 1 out of every 4 deaths in the U.S and $16 \%$ of the world's total deaths $(1,2)$. With the global population of persons aged 65 and older increasing each year, the prevalence of heart disease is also increasing (3). Among those with heart disease, heart failure, a serious condition, afflicts more than 6.2 million American adults and accounts for $13.4 \%$ of deaths each year, more than half of all heart disease attributable deaths (4). The number of American adults with heart failure is estimated to increase to more than 8 million, or about $3 \%$ of the population, by 2030 (4). Overall lifetime risk of developing heart failure ranges from $20 \%$ at age 45 to $45 \%$ at age 95 , with variability between sex and race. There are also significant increases in lifetime risk among those with certain risk factors such as elevated blood pressure and body mass index (4).

The burden of heart failure is expansive, with high morbidity and mortality compared to many other chronic diseases. Heart failure results in decreased quality of life, disability, financial hardship, increased medical expenses, and more frequent hospital visits. The total economic cost of heart failure in the U.S. is projected to reach $\$ 70$ billion by 2030 with more than two-thirds of the cost in direct medical expenses (4). Heart failure is one of the leading causes for in-patient hospitalization stays in the U.S with more than 1 million each year (5). The outcomes of heart failure patients after hospitalization are poor with case fatality rates of $10.4 \%$ at 30 days, $22 \%$ at 1 year, and $42.3 \%$ after 5 years, according to the National Heart, Lung, and Blood Institute's Atherosclerosis Risk in Communities study (4).

Several types of heart failure have been identified based on the specific cardiac dysfunctional characteristics of the patient. The subset of heart failure classified as HFpEF accounts for at least $50 \%$ of heart failure cases and is more commonly diagnosed in women. Current treatment options for $\mathrm{HFpEF}$ are limited to mainly symptom management, heart failure risk factor reduction, and comorbid disease treatments. With the expected increasing prevalence of HFpEF cases in an aging population, new treatments targeted specifically for this disease are needed to improve patient outcomes and quality of life.

Mitochondrial dysfunction may play a significant role in the pathogenesis of HFpEF (6). A primary characteristic of mitochondrial dysfunction is impaired synthesis of adenosine triphosphate (ATP), the cellular energy molecule. Without an adequate supply of ATP, cardiomyocytes may not be able to function normally, initiating or contributing to symptoms of heart failure. D-ribose is a naturally and endogenously generated monosaccharide critical for cellular energy production. Emerging evidence shows promise for the supplemental use of D-ribose to manage heart failure symptoms.

\section{Objective}

This article explores the role that supplemental D-ribose may have for patients with HFpEF, including possible benefits, disadvantages, and mechanisms of action. Key questions addressed in this review include the following:

(I) What is Heart Failure with Preserved Ejection Fraction?

(II) How does mitochondrial bioenergetics affect HFpEF?

(III) What role does supplemental D-ribose have for HFpEF treatment?

We present the following article in accordance with the Narrative Review reporting checklist (available at https:// dx.doi.org/10.21037/atm-21-2291).

\section{HFpEF}

Heart failure can be classified in several ways (acute $v s$. chronic, left $v s$. right sided, forward $v s$. backwards, and low vs. high output). One of the most common and useful classifications is by ejection fraction. Heart failure with reduced ejection fraction ( $\mathrm{HFrEF}$ ), is also called systolic heart failure. With this form of heart failure, the left ventricle (LV) loses its ability to contract normally and left ventricular ejection fraction is less than $40 \%$. In contrast, $\mathrm{HFpEF}$ is also called diastolic heart failure in which the diastolic function of the $\mathrm{LV}$ is impaired due to a stiff or noncompliant LV. The heart is unable to adequately fill with blood during the diastolic period between each contraction. $\mathrm{HFpEF}$ is characterized by signs and symptoms of heart failure and a left ventricular ejection fraction (LVEF) $\geq 50 \%$. Heart failure associated with intermediate reductions in LVEF ( $40 \%$ to $49 \%$ ) is also commonly grouped into a new category called heart failure with midrange failure (HFmrEF). HFpEF causes almost one-half of the more than 6 million cases of heart failure in the U.S. (7). It is more common among older patients, obese women, and those with hypertension (8). HFpEF results from abnormalities 
Table 1 Causes of HFpEF

Anemia
Atrial fibrillation
Chronic kidney disease
Chronic obstructive pulmonary disease (COPD)
Coronary artery disease
Diabetes mellitus
Hypertension
Hypertrophic cardiomyopathy
Obesity
Obstructive sleep apnea
Pericardial disease
Valvular heart disease

HFpEF, heart failure with preserved ejection fraction.

of active ventricular relaxation and is often caused by hypertension, coronary artery disease, and valvular disease (Table 1). The prognosis of HFpEF is comparable to that of $\mathrm{HFrEF}$ and is worsened by higher levels of brain natriuretic peptide, older age, a history of myocardial infarction, and significantly reduced diastolic function.

HFpEF should be suspected in patients with typical symptoms (fatigue, weakness, dyspnea, orthopnea, and paroxysmal nocturnal dyspnea) and signs (S4 heart sound, edema, rales, and jugular venous distension) of chronic heart failure. Echocardiographic findings of normal ejection fraction with impaired diastolic function confirm the diagnosis. The echocardiographic criteria recommended by the European Society of Cardiology for the diagnosis of HFpEF includes a LVEF of $\geq 50 \%$ and LV end-diastolic volume index (LVEDI) $<97 \mathrm{~mL} / \mathrm{m}^{2}$ (9). Elevated $\mathrm{LV}$ filling pressure is indicated by a ratio of mitral early diastolic inflow velocity to mitral early annular lengthening velocity $\left(\mathrm{E} / \mathrm{e}^{\prime}\right)>15$ (10). Measurement of natriuretic peptides is also useful in the evaluation of the severity of the disease in patients with HFpEF (11).

Clinical trials of pharmacologic therapy for HFpEF have produced largely neutral results (12). In the absence of definitive evidence, current management strategies should be based on an understanding of the underlying pathophysiologic processes in HFpEF. Thus, the management of patients with HFpEF is principally directed toward treating associated or contributing conditions (such as coronary artery disease, hypertension, or atrial fibrillation) and symptoms (edema and other findings of congestion or volume overload). This approach is consistent with recommendations for treatment of patients with $\mathrm{HFpEF}$ included in the 2017 focused update of the 2013 American College of Cardiology Foundation/ American Heart Association (ACC/AHA) Heart Failure guidelines (13). Management of heart failure should be provided according to published clinical practice guidelines to improve symptomatic heart failure.

Multiple trials have not demonstrated medications to be effective treatment of the underlying physiology of HFpEF, except for palliative use of diuretics. Patients with congestive symptoms should be treated with a diuretic to reduce volume overload. If hypertension is present, it should be treated according to evidence-based guidelines. It is important to note that clinical trials of angiotensin receptor blockers raise concerns about adverse effects, and these medications should be used with caution. Exercise and treatment by multidisciplinary teams may be helpful. Physicians should consider referring patients with HFpEF who can exercise safely for exercise training or cardiac rehabilitation.

Studies have shown that in HFpEF there is muscle mitochondrial energetic impairment, particularly during exertion $(14,15)$. There are still questions on the pathogenesis that leads to this mitochondrial dysfunction including oxidative stress, changes in sympathetic tone, increased cytokine production, insulin resistance etc. There are also differences in mitochondrial dysfunction between patients with HFpEF and HFrEF. In HFpEF patients, there is an increase in impairment of mitochondria function resulting in less ATP production $(16,17)$.

Currently, there are limited treatment options for HFpEF management, and the large patient population impacted by this disorder has an urgent need for effective and safe therapies. Due to their effects on mitochondrial bioenergetics, supplemental D-ribose and ubiquinol could be potential options for HFpEF management. Providing evidence-based recommendations for management alternatives of this debilitating and common syndrome is of vital importance.

\section{Mitochondria and bioenergetics}

Mitochondria are important organelles that are responsible for cellular energy production. They are composed of inner and outer membranes with an intermembrane space between the two membranes. The proteins within the outer membrane are called porins and they are essential for 
movement of ions in and out of the mitochondrion. The cristae space is the inner membrane of the mitochondria generating a high surface area that allows for increased ATP generation. Cristae are studded with many proteins (i.e., ATP synthase, complexes I-IV) that are involved in electron transport, ATP synthesis, and transport of metabolites in and out of the mitochondrial matrix (18). There are also other mitochondrial components such as granules, ribosomes, and mitochondrial deoxyribonucleic acid (mtDNA). The mtDNA play an important role in the regulation of apoptosis, free radical generation, and cellular metabolism (19).

Mitochondria produce cellular energy through the process of respiration and regulate cellular metabolism $(20,21)$. Mitochondria are central to the synthesis of adenosine triphosphate (ATP) (22). ATP consists of three principal structures: (I) adenine, the nitrogenous base; (II) ribose, the sugar; and (III) phosphate groups connected to ribose (23). ATP is synthesized by the enzyme ATP synthase and it is an important and essential cellular energy source for the myocardium (24,25). Mitochondria participate in three major cellular processes, including: (I) oxidative phosphorylation which is the synthesis of ATP by phosphorylation for energy via the electron transport chain; (II) cellular respiration where glycolysis occurs; and (III) bioenergetics where cellular energy transformations and transductions arise (26-28). The electron transport chain consists of a series of protein complexes (I-IV) that contains enzymes, peptides, and many other molecules. During this process there is a transfer of electrons that are involved in moving electrons from $\mathrm{NADH}$ and $\mathrm{FADH}_{2}$ to molecular oxygen. The protons are moved from the mitochondrial matrix to the intermembrane space allowing oxygen to be reduced to water (29). Under normal conditions about 4\% of ATP is generated by glycolysis and $1 \%$ from the citric acid cycle. Approximately $95 \%$ of ATP is generated in the myocardium derived from oxidative phosphorylation in the mitochondria (30).

Myocardial mitochondria are the main source of energy for cardiac metabolism. These organelles are very dynamic with quality control measures to enhance performance of the muscle. One of the major pathophysiologic mechanisms now recognized for heart failure is mitochondrial dysfunction. Under physiologic conditions, mitochondria play a major role in cardiomyocyte energy production, electrolyte homeostasis, apoptosis, and calcium signaling. Since the mitochondrial respiratory chain produces oxygenfree radicals, the inner and outer membranes assist mtDNA in damage repair and communication among organelles. Furthermore, the genome within the nucleus regulates the replication of mtDNA and the synthesis of mitochondrial proteins (31).

In patients with $\mathrm{HFpEF}$, there are structural and energetic cardiomyocyte mitochondria aberrations. These changes may occur from different pathophysiologic mechanisms such as increased free radical damage to the mitochondria that leads to reduced ATP production (30). With the alteration in supply and demand of ATP to the cardiomyocyte, there is often activation of downstream signaling pathways causing inflammation and diastolic dysfunction (32). These changes in mitochondrial function are now being included in the pathophysiology of HFpEF (17).

\section{Cellular D-ribose}

D-ribose is a naturally occurring 5 -carbon monosaccharide (pentose) that serves an important role in the genetic and energetic structures within the cell (33). D-ribose exists in equilibrium as an open-chain and 2-ring structures. The open chain structure of D-ribose is an aldose due to it containing an aldehyde functional group on $\mathrm{C} 1$. The cyclic forms of D-ribose are the pentagonal ring structure (the furanose) from the reaction with the aldehyde and $\mathrm{C} 4$, and the hexagonal ring structure (the pyranose) from the reaction of the aldehyde and C5. Both ring structures have $\alpha$ and $\beta$ forms depending on the position of the constituents attached to C1 (34). The furanose ring structure of D-ribose is a precursor to the structures of nucleic acids (DNA and RNA), coenzymes (NADH, $\mathrm{NADPH}, \mathrm{FADH}_{2}$, and acetyl coenzyme A), and energy molecules (ATP, GTP, etc.) $(33,35)$.

$\mathrm{D}$-ribose is synthesized in the cell's cytosol by the pentose phosphate pathway (PPP) (33). This pathway is also known as the hexose monophosphate shunt (HMS) due to it "shunting" glucose-6-phosphate (G6P) from other metabolic pathways to the PPP. Since G6P is used in other pathways such as glycolysis, gluconeogenesis, and glycogenesis, the first step in the PPP is regulated by glucose-6-phosphate dehydrogenase (G6PDH), an enzyme that is stimulated by increasing levels of $\mathrm{NADP}^{+}$ $(35,36)$. The first half of the PPP is considered the oxidative phase. G6P is oxidized by G6PDH with $\mathrm{NADP}^{+}$ to form D-6-phosphogluconolactone and NADPH. D-6phosphogluconolactone is further oxidized forming more $\mathrm{NADPH}$, hydrolyzed, and decarboxylated to form ribulose5-phospate (37). The NADPH produced from this pathway 


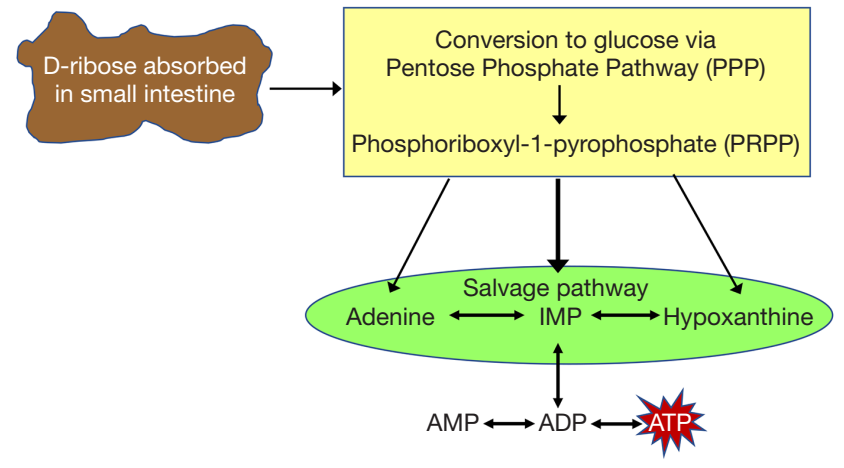

Figure 1 There is a decrease in the intracellular myocardium concentrations of total adenine nucleotides with heart failure and the body replaces ATP by using either the salvage or de novo pathways of purine nucleotide metabolism. This reformative process starts the conversion of glucose through the pentose phosphate pathway (PPP). During this process phosphoribosyl1-pyrophosphate (PRPP) is converted to inosine monophosphate (IMP) then to adenosine monophosphate (AMP). Once there is more AMP, it can be converted to ATP for energy (40).

provides the cells with antioxidant defense and participates in anabolic reactions for cholesterol, steroids, and fatty acids (36). The second half of the PPP is the non-oxidative phase. The ribulose-5-phosphate formed from the first phase undergoes non-oxidative reactions that form the key components: ribose-5-phosphate (R5P), fructose-6phosphate (F6P), and glyceraldehyde-3-phosphate (G3P). F6P and G3P can feedback into glycolysis and contribute to the production of ATP and intermediates for cellular respiration (37). R5P contributes to the synthesis of nucleotides by undergoing a double phosphorylation from an ATP molecule to produce an activated form called phosphoribosyl pyrophosphate (PRPP). PRPP can enter two pathways in order to synthesize ribonucleotides for RNA and ATP. The salvage pathway recycles existing nitrogenous bases and adds them to PRPP. The de novo pathway uses simple molecules such as amino acids to create the nitrogenous bases that attaches to the pentose ring $(35,38,39)$. $\mathrm{NAD}^{+}$and NADP can be synthesized from PRPP by the salvage pathway with nicotinamide and the de novo pathway with tryptophan (40). In patients with HFpEF, there is decreased intracellular myocardium adenine nucleotide concentrations. Ingestion of D-ribose allows for conversion of glucose through the PPP, PRPP, and salvage pathways into ATP (Figure 1) (41).

The PPP is a slow and rate-limited process due to the limited availability of G6PDH (42). D-ribose plays an important role in providing a framework for the formation of molecules that transfer and produce energy and the production of intermediates that can feedback into other pathways for cellular respiration. Researchers have increasing interest in exploring the possibility of supplemental D-ribose as a means to increase intracellular energy in cells with impaired bioenergetics (33). Supplemental D-ribose bypasses the oxidative phase of the PPP by first being phosphorylated by a ribokinase and forming $\mathrm{R} 5 \mathrm{P}$ that feeds into the nonoxidative phase of the PPP (34). Even though supplemental D-ribose may consume energy during phosphorylation and omit the formation of NADPH from the oxidative phase, it is hypothesized that supplemental D-ribose can contribute an increase in overall cellular energy by accelerating the synthesis of ATP $(42,43)$. It is thought that D-ribose may benefit patients with mitochondrial dysfunction by increasing ATP levels. A study by Omran et al. evaluated the effect of supplemental D-ribose on diastolic function in patients with heart failure. The study found significant echocardiographic data indicating an improvement in diastolic function and significant enhancement in perceived quality of life from the questionnaires completed by participants in the D-ribose group (22). Various studies have assessed the use of supplemental D-ribose, but all have been limited by either a small sample size, a lack of diversity in subject demographics, varying fitness levels of participants in studies observing the effect on D-ribose and exercise, absent monitoring of diets that may contain D-ribose, dosing amount of D-ribose, or varying duration of supplementation (44-46). D-ribose may have an overall benefit, but some studies have shown that D-ribose may participate in protein glycation leading to cell cytotoxicity (34). Glycation can cause the production of reactive oxygen species (ROS) and advanced glycation end-products (AGEs) that can accumulate and form protein aggregates. AGEs have been associated with aging and neurodegenerative diseases (47). In vitro studies using human serum albumin (HSA) and in vivo studies using mice models have shown the ability of D-ribose to glycate proteins leading to glycated serum proteins. Further research is needed on the role of supplemental D-ribose on the possible formation of AGEs by protein glycation in humans (48).

\section{HFpEF and use of D-ribose}

Mitochondrial function requires nutrients and oxygen for metabolic function, especially for myocardial tissue. Mitochondria can be damaged from many sources 
such as pharmaceutical drugs, cigarette smoke, genetic abnormalities, occupational chemicals, etc. Patients with cardiovascular disease where mitochondrial dysfunction occurs can cause a progressive decline in ATP synthesis. When decline of ATP in mitochondria arises, there is increased oxidative stress with loss of structural integrity of mitochondria (20). This mitochondrial dysfunction from ATP depletion and free radical damage occurs in many organs and leads to various diseases.

Normal myocardium produces approximately 50 $70 \%$ of the ATP for mitochondrial $\beta$-oxidation of fatty acids. In patients with HFpEF, there is a diminishing in mitochondrial bioenergetics that leads to reduced myocardial ATP. This decrease in APT production in $\mathrm{HFpEF}$ results in diminished cardiac function and performance. The myocardial mitochondria will shift from using lipids to glucose in an attempt to maintain ATP production for cellular function. Often in patients with $\mathrm{HFpEF}$, this mechanism is disrupted and there is significant loss of ATP leading to apoptosis of cardiomyocytes. The respiratory chain is also altered and there is decreased activity within the mitochondrial complexes I thru V leading to less ATP production. With heart failure, the replication of mtDNA is severely diminished which causes a decrease in mtDNA-encoded proteins. Without these proteins, the mitochondrial biogenesis (mass) is impaired. Myocardial cells control the rate of mitochondrial biogenesis through SIRT1-dependent pathways that reduce myocardial oxidative stress and prevent apoptosis $(20,21)$. There are several studies that have demonstrated that mitochondrial dysfunction plays a major role in the pathogenesis of heart failure (49) (Table 2).

Supplemental D-Ribose has been investigated in animal models to enhance myocardial metabolism and performance following myocardial ischemia $(23,24)$. It has also been used with humans to examine the effects of oral D-ribose supplementation on cardiac hemodynamics and quality of life. In this study the participants consumed D-ribose for 3 weeks and a placebo for 3 weeks. The investigators found improved diastolic function and enhanced quality of life indicators with D-ribose but not with the placebo powder (22). Derosa et al. investigated 53 ischemic heart disease patients and tested several nutraceutical compounds. These include D-ribose with creatine, vitamin $B_{1}$, and vitamin $B_{6}$. They concluded that these supplements with a physical exercise program, improved their exercise tolerance (27). Another study examined 11 patients with heart failure (New York Heart
Association class II-IV) that had clinical symptoms, normal left ventricular systolic function, and diastolic dysfunction. The patients received 5 grams of D-ribose daily for 6 weeks. They found improvement in diastolic filling velocity and maximal oxygen consumption (28). Thus, supplemental D-ribose is a pentose carbohydrate that helps replenish deficient ATP levels in patients with HFpEF. With the use of D-ribose, the cells are able to bypasses a key metabolic enzyme needed for the production of ATP and assist the failing depleted myocardium heart to produce energy and reduce diastolic dysfunction $(28,57)$. These studies suggest that $\mathrm{D}$-ribose could be a potential treatment for patients with HFpEF or diastolic dysfunction.

\section{Methods}

This review considered research articles and systematic reviews of HFpEF, D-ribose, mitochondrial bioenergetics, cellular respiration, and ATP production from January 2011 to April 2021. Inclusion of systematic review articles ensured that we maximized the examination of studies that may not clearly use the terms HFpEF, bioenergetics, and cellular respiration. The literature search was completed using the following strategy. With collaboration of a research team, literature searches were conducted in the databases PubMed, Web of Science, CINAHL plus Full Text, and Google Scholar from January 2011 to April 2021. For this search, keywords for the concepts of "HFpEF", "Heart Failure", "D-ribose", "ATP", "Mitochondria", Bioenergetics" and "Cellular Respiration" were combined in varied ways using "AND" and "OR" search operators. Limits were applied to the search including articles published in the last 10 years and when available, humans and RCT filters. A PubMed search using only medical subject headings $(\mathrm{MeSH})$ was conducted to ensure retrieval of the most precise results. Although certain limitations were found in some of the studies due to sample size, the overall trend of using D-ribose supplementation for HFpEF was positive.

\section{Discussion}

D-Ribose has been shown in animal and human studies to increase myocardial ATP production and improve cardiac function. In a few clinical trials, oral D-ribose supplementation enhanced cardiac hemodynamics, ejection fraction, and quality of life in patients with heart failure. 
Table 2 Pre-clinical and clinical articles related to the D-ribose and heart disease

\begin{tabular}{|c|c|}
\hline Articles & Summary \\
\hline $\begin{array}{l}\text { Ojetola, Adedeji, \& Fasanmade, } \\
\text { (2021) (50) }\end{array}$ & $\begin{array}{l}\text { This study investigated cardiovascular effects of different doses of D-Ribose-L-cystein (DRLC) Wistar } \\
\text { rats. Prolonged administration of DRLC } 250 \mathrm{mg} / \mathrm{kg} \text { supplementation, increased possess the ability to } \\
\text { yield better cardiovascular responses. Thus, suggesting that supplementation with DRLC at } \\
250 \mathrm{mg} / \mathrm{kg} \text { may improve cardiac function by decreasing in triglycerides, atherogenic index and } \\
\text { C-reactive protein }\end{array}$ \\
\hline $\begin{array}{l}\text { St Cyr, Bianco, Schneider, } \\
\text { Mahoney, Tveter, \& Einzig } \\
\text { (1989) (23) }\end{array}$ & $\begin{array}{l}\text { In canines, these investigators found that d-ribose enhance recovery of diastolic function follow } \\
20 \text { minutes of induced myocardial ischemia }\end{array}$ \\
\hline $\begin{array}{l}\text { Li, Wang, Xiao, Zhang, Fang, } \\
\text { Yang, Zhang, Masser, Qin } \\
\text { (2021) (52) }\end{array}$ & $\begin{array}{l}\text { Review article of the potential physiological functions of D-ribose, its toxic effects, clinical value and } \\
\text { its utility for the treatment of heart failure and diabetes }\end{array}$ \\
\hline $\begin{array}{l}\text { Pierce, Shen, Vacek, Rahman, } \\
\text { Krueger, Gupta, \& Hiebert } \\
\text { (2020) (35) }\end{array}$ & $\begin{array}{l}\text { A review article concerning } d \text {-ribose and how it may improve mitochondrial bioenergetics. } \\
\text { It specifically addresses the role of mitochondrial metabolism as it may relate to HFpEF } \\
\text { pathophysiology and the potential mechanisms by which ubiquinol and d -ribose may impact } \\
\text { mitochondrial function }\end{array}$ \\
\hline $\begin{array}{l}\text { Derosa, Pasqualotto, Catena, } \\
\text { D’Angelo, \& Maffioli, (2019) (27) }\end{array}$ & $\begin{array}{l}\text { A clinical trial examining } 53 \text { subjects who were given a nutraceutical composition containing creatine, } \\
\text { D-ribose, vitamin B1, and vitamin B6 or the placebo. These supplements in addition to standard } \\
\text { therapy and exercise improved exercise tolerance I patients with cardiovascular disease }\end{array}$ \\
\hline $\begin{array}{l}\text { Bayram, St. Syr, \& Abraham } \\
(2015)(27)\end{array}$ & $\begin{array}{l}\text { In } 11 \text { patients with heart failure, oral D-ribose was administered for } 6 \text { weeks. These investigators } \\
\text { found that D-ribose improved cardiac function measured by echocardiogram in patients with } \\
\text { diastolic heart failure }\end{array}$ \\
\hline $\begin{array}{l}\text { Wagner, Herrick, Shecterle, \& St. } \\
\text { Cyr (2009) (55) }\end{array}$ & $\begin{array}{l}\text { This review article outlined studies that indicate that D-ribose may improve diastolic dysfunction } \\
\text { following myocardial ischemia, and congestive heart failure. They suggest that D-ribose provides the } \\
\text { necessary metabolic substrate during heart failure when the myocardium is in an energy-deficient } \\
\text { state }\end{array}$ \\
\hline Herrick \& St. Cyr (2008) (56) & $\begin{array}{l}\text { In this article, the authors review states of ischemia and/or hypoxia and the lower levels of cellular } \\
\text { myocardial energy. They propose that ribose appears to provide a potential solution to the problem } \\
\text { in replenishing ATP levels in patients with cardiovascular diseases }\end{array}$ \\
\hline $\begin{array}{l}\text { Omran, Illien, MacCarter, St. Cyr, } \\
\text { Luderitz, (2003) (22) }\end{array}$ & $\begin{array}{l}\text { In this feasibility study, } 15 \text { subjects with chronic coronary artery disease and heart failure were given } \\
\text { oral D-ribose or placebo. After } 3 \text { weeks of D-ribose, the data indicated patients with coronary artery } \\
\text { disease and heart failure had improved diastolic function and quality of life }\end{array}$ \\
\hline
\end{tabular}

HFpEF, heart failure with preserved ejection fraction. 
Unfortunately, the major limitation of these studies was the small sample size of 15 or less participants. Also, there was no measurement in these studies of ATP concentration or mitochondria. On clinicaltrials.gov, a recently completed study with HFpEF patients has been completed with results indicating that oral supplemental D-ribose increased ATP production and improved ejection fraction. Improving myocardial ATP deficiency by enhancing mitochondrial bioenergetics could offer a potential treatment for patients with HFpEF.

There are also data measuring mitochondrial proteins that are continually remodeling to improve mitochondrial function (58). This constant synthesis and turnover of mitochondrial proteins are more susceptible to free radical damage affecting oxidation phosphorylation and ultimately ATP production (59). Thus, supplemental D-ribose may be able to assist with maintaining myocardial energy production and reduce the damage to mitochondrial transcriptome and proteome allowing for improved diastolic function (52).

With HFpEF there are decreased concentrations of ATP in cardiac myocytes, and to preserve adequate cardiac ATP supplies ADP/ATP ratio must be held as low as possible. If the ratio increases, there can be cardiac dysfunction caused by a limited ability to convert ADP to ATP in the circulation. Thus, supplemental D-ribose may accelerate PRPP synthesis directly to increase myocardial ATP production and decrease HFpEF. Chen et al. found that there was a time-dependent association between ATP concentration and diastolic function. When D-ribose was administered, there was a quick restoration of ATP and reduction of diastolic dysfunction (60). Thus, D-ribose improves function and limits damage through bypassing the rate-limiting step in the PPP pathway causing a rise in PRPP by increasing the de novo synthesis rate of ATP (Figure 1) (52).

There are many early-phase clinical trials focusing on mitochondrial agents for patients with HFpEF. These therapeutic approaches include strategies for modulation of mitochondrial function, particularly stimulation of mitochondrial biogenesis. D-ribose, ubiquinol (CoQ10), and resveratrol have been studied in HFpEF with a completed study assessing the effects of these agents on cardiac function; however, the results have not yet been published (17).

\section{Conclusions}

HFpEF is a debilitating disease with few effective medical treatment options for patients. With mitochondrial dysfunction in HFpEF patients, ATP synthesis is impaired, potentially presenting with symptoms of fatigue and shortness of breath. D-ribose supplementation may support mitochondrial energy production through acceleration of ATP synthesis by means of bypassing the oxidative phase of the pentose phosphate pathway and resulting in an increased quantity of available intracellular ATP. The mitochondrial bioenergetic pathways suggest that supplemental D-ribose may be a viable therapeutic option for HFpEF patients.

\section{Acknowledgments}

Funding: This work was supported by the Department of Health and Human Services, National Institutes of Health, National Institute on Aging [Grant Number: 1R01AG054486-01A1].

\section{Footnote}

Reporting Checklist: The authors have completed the Narrative Review reporting checklist. Available at https:// dx.doi.org/10.21037/atm-21-2291

Conflicts of Interest: All authors have completed the ICMJE uniform disclosure form (available at https://dx.doi. org/10.21037/atm-21-2291). Dr. KJK, Dr. JBH, and Dr. JDP reported funding received from NIH R01 grant for clinical trial. The other authors have no conflicts of interest to declare.

Ethical Statement: The authors are accountable for all aspects of the work in ensuring that questions related to the accuracy or integrity of any part of the work are appropriately investigated and resolved.

Open Access Statement: This is an Open Access article distributed in accordance with the Creative Commons Attribution-NonCommercial-NoDerivs 4.0 International License (CC BY-NC-ND 4.0), which permits the noncommercial replication and distribution of the article with the strict proviso that no changes or edits are made and the original work is properly cited (including links to both the formal publication through the relevant DOI and the license). See: https://creativecommons.org/licenses/by-nc-nd/4.0/.

\section{References}

1. Ahmad FB, Anderson RN. The Leading Causes of Death 
in the US for 2020. JAMA 2021;325:1829-30.

2. Roth GA, Mensah GA, Johnson CO, et al. Global Burden of Cardiovascular Diseases and Risk Factors, 1990-2019: Update From the GBD 2019 Study. J Am Coll Cardiol 2020;76:2982-3021.

3. Khan MA, Hashim MJ, Mustafa H, et al. Global Epidemiology of Ischemic Heart Disease: Results from the Global Burden of Disease Study. Cureus 2020;12:e9349.

4. Virani SS, Alonso A, Benjamin EJ, et al. Heart Disease and Stroke Statistics-2020 Update: A Report From the American Heart Association. Circulation 2020;141:e139-596.

5. HCUP Fast Stats - Healthcare Cost and Utilization Project (HCUP) Rockville, MD: Agency for Healthcare Research and Quality 2021 [updated April 2021. Available online: www.hcup-us.ahrq.gov/faststats/national/ inpatientcommondiagnoses.jsp?year $1=2018 \&$ characteristic $1=0$ \&included $1=0$ \&year $2=\&$ characteristic $2=0$ \&included $2=$ 1\&expansionInfoState=hide\&dataTablesState=hide\&defini tionsState=hide $\&$ exportState $=$ hide .

6. Yousefi K, Irion CI, Takeuchi LM, et al. Osteopontin Promotes Left Ventricular Diastolic Dysfunction Through a Mitochondrial Pathway. J Am Coll Cardiol 2019;73:2705-18.

7. Simmonds SJ, Cuijpers I, Heymans S, et al. Cellular and Molecular Differences between HFpEF and HFrEF: A Step Ahead in an Improved Pathological Understanding. Cells 2020;9:242.

8. Ekström M, Hellman A, Hasselström J, et al. The transition from hypertension to hypertensive heart disease and heart failure: the PREFERS Hypertension study. ESC Heart Fail 2020;7:737-46.

9. Henning RJ. Diagnosis and treatment of heart failure with preserved left ventricular ejection fraction. World $\mathrm{J}$ Cardiol 2020;12:7-25.

10. Andersen OS, Smiseth OA, Dokainish H, et al. Estimating Left Ventricular Filling Pressure by Echocardiography. J Am Coll Cardiol 2017;69:1937-48.

11. Selvaraj S, Kim J, Ansari BA, et al. Body Composition, Natriuretic Peptides, and Adverse Outcomes in Heart Failure With Preserved and Reduced Ejection Fraction. JACC Cardiovasc Imaging 2021;14:203-15.

12. Palazzuoli A, Caravita S, Paolillo S, et al. Current gaps in HFpEF trials: Time to reconsider patients' selection and to target phenotypes. Prog Cardiovasc Dis 2021;67:89-97.

13. Yancy CW, Jessup M, Bozkurt B, et al. 2017 ACC/ AHA/HFSA Focused Update of the 2013 ACCF/AHA Guideline for the Management of Heart Failure: A Report of the American College of Cardiology/American Heart Association Task Force on Clinical Practice Guidelines and the Heart Failure Society of America. J Am Coll Cardiol 2017;70:776-803.

14. Kitzman DW, Higginbotham MB, Cobb FR, et al. Exercise intolerance in patients with heart failure and preserved left ventricular systolic function: failure of the Frank-Starling mechanism. J Am Coll Cardiol 1991;17:1065-72.

15. Bowen TS, Rolim NP, Fischer T, et al. Heart failure with preserved ejection fraction induces molecular, mitochondrial, histological, and functional alterations in rat respiratory and limb skeletal muscle. Eur J Heart Fail 2015;17:263-72.

16. Weiss K, Schär M, Panjrath GS, et al. Fatigability, Exercise Intolerance, and Abnormal Skeletal Muscle Energetics in Heart Failure. Circ Heart Fail 2017;10:e004129.

17. Kumar AA, Kelly DP, Chirinos JA. Mitochondrial Dysfunction in Heart Failure With Preserved Ejection Fraction. Circulation 2019;139:1435-50.

18. Miranda-Silva D, Lima T, Rodrigues P, et al. Mechanisms underlying the pathophysiology of heart failure with preserved ejection fraction: the tip of the iceberg. Heart Fail Rev 2021;26:453-78.

19. Sharma P, Sampath H. Mitochondrial DNA Integrity: Role in Health and Disease. Cells 2019;8:100.

20. Chistiakov DA, Shkurat TP, Melnichenko AA, et al. The role of mitochondrial dysfunction in cardiovascular disease: a brief review. Ann Med 2018;50:121-7.

21. Siasos G, Tsigkou V, Kosmopoulos M, et al. Mitochondria and cardiovascular diseases-from pathophysiology to treatment. Ann Transl Med 2018;6:256.

22. Omran H, Illien S, MacCarter D, et al. D-Ribose improves diastolic function and quality of life in congestive heart failure patients: a prospective feasibility study. Eur J Heart Fail 2003;5:615-9.

23. St Cyr JA, Bianco RW, Schneider JR, et al. Enhanced high energy phosphate recovery with ribose infusion after global myocardial ischemia in a canine model. J Surg Res 1989;46:157-62.

24. Ward HB, St Cyr JA, Cogordan JA, et al. Recovery of adenine nucleotide levels after global myocardial ischemia in dogs. Surgery 1984;96:248-55.

25. Pauly DF, Pepine CJ. D-Ribose as a supplement for cardiac energy metabolism. J Cardiovasc Pharmacol Ther 2000;5:249-58.

26. Illien S, Omran H, MacCarter D, St Cyr JA. Ribose improves myocardial function in congestive heart failure. FASEB J 2001;15:A1142. 
27. Derosa G, Pasqualotto S, Catena G, et al. A Randomized, Double-Blind, Placebo-Controlled Study to Evaluate the Effectiveness of a Food Supplement Containing Creatine and D-Ribose Combined with a Physical Exercise Program in Increasing Stress Tolerance in Patients with Ischemic Heart Disease. Nutrients 2019;11:3075.

28. Bayram M, St Cyr JA, Abraham WT. D-ribose aids heart failure patients with preserved ejection fraction and diastolic dysfunction: a pilot study. Ther Adv Cardiovasc Dis 2015;9:56-65.

29. Lin KL, Chen SD, Lin KJ, et al. Quality Matters? The Involvement of Mitochondrial Quality Control in Cardiovascular Disease. Front Cell Dev Biol 2021;9:636295.

30. Doenst T, Nguyen TD, Abel ED. Cardiac metabolism in heart failure: implications beyond ATP production. Circ Res 2013;113:709-24.

31. Qiu Z, Wei Y, Song Q, et al. The Role of Myocardial Mitochondrial Quality Control in Heart Failure. Front Pharmacol 2019;10:1404.

32. Dai DF, Chen T, Szeto H, et al. Mitochondrial targeted antioxidant Peptide ameliorates hypertensive cardiomyopathy. J Am Coll Cardiol 2011;58:73-82.

33. Mahoney DE, Hiebert JB, Thimmesch A, et al. Understanding D-Ribose and Mitochondrial Function. Adv Biosci Clin Med 2018;6:1-5.

34. Del-Corso A, Cappiello M, Moschini R, et al. The furanosidic scaffold of d-ribose: a milestone for cell life. Biochem Soc Trans 2019;47:1931-40.

35. Pierce JD, Shen Q, Vacek J, et al. Potential use of ubiquinol and d-ribose in patients with heart failure with preserved ejection fraction. Ann Med Surg (Lond) 2020;55:77-80.

36. Akram M, Ali Shah SM, Munir N, et al. Hexose monophosphate shunt, the role of its metabolites and associated disorders: A review. J Cell Physiol 2019. [Epub ahead of print]. doi: 10.1002/jcp.28228.

37. Stincone A, Prigione A, Cramer T, et al. The return of metabolism: biochemistry and physiology of the pentose phosphate pathway. Biol Rev Camb Philos Soc 2015;90:927-63.

38. Berg JM TJ, Stryer L. Nucleotide Biosynthesis. Biochemistry. 5th ed. New York: W. H. Freeman and Company; 2002.

39. Hove-Jensen B, Andersen KR, Kilstrup M, et al. Phosphoribosyl Diphosphate (PRPP): Biosynthesis, Enzymology, Utilization, and Metabolic Significance. Microbiol Mol Biol Rev 2017;81:e00040-16.
40. Xiao W, Wang RS, Handy DE, et al. NAD $(\mathrm{H})$ and NADP(H) Redox Couples and Cellular Energy Metabolism. Antioxid Redox Signal 2018;28:251-72.

41. Dodd SL, Johnson CA, Fernholz K, et al. The role of ribose in human skeletal muscle metabolism. Med Hypotheses 2004;62:819-24.

42. Thompson J, Neutel J, Homer K, et al. Evaluation of D-ribose pharmacokinetics, dose proportionality, food effect, and pharmacodynamics after oral solution administration in healthy male and female subjects. J Clin Pharmacol 2014;54:546-54.

43. Pierce JD, Mahoney DE, Hiebert JB, et al. Study protocol, randomized controlled trial: reducing symptom burden in patients with heart failure with preserved ejection fraction using ubiquinol and/or D-ribose. BMC Cardiovasc Disord 2018;18:57.

44. Cao W, Qiu J, Cai T, et al. Effect of D-ribose supplementation on delayed onset muscle soreness induced by plyometric exercise in college students. J Int Soc Sports Nutr 2020;17:42.

45. Kreider RB, Melton C, Greenwood M, et al. Effects of oral D-ribose supplementation on anaerobic capacity and selected metabolic markers in healthy males. Int J Sport Nutr Exerc Metab 2003;13:76-86.

46. Seifert JG, Brumet A, St Cyr JA. The influence of D-ribose ingestion and fitness level on performance and recovery. J Int Soc Sports Nutr 2017;14:47.

47. Wei Y, Han CS, Zhou J, et al. D-ribose in glycation and protein aggregation. Biochim Biophys Acta 2012;1820:488-94.

48. Chen Y, Yu L, Wang Y, et al. d-Ribose contributes to the glycation of serum protein. Biochim Biophys Acta Mol Basis Dis 2019;1865:2285-92.

49. Bisaccia G, Ricci F, Gallina S, et al. Mitochondrial Dysfunction and Heart Disease: Critical Appraisal of an Overlooked Association. Int J Mol Sci 2021;22:614.

50. Ojetola AA, Adedeji TG, Fasanmade AA. Changes in antioxidants status, atherogenic index and cardiovascular variables after prolonged doses of D-ribose-L-cysteine in male Wistar rats. Heliyon 2021;7:e06287.

51. Lamberts RR, Caldenhoven E, Lansink M, et al. Preservation of diastolic function in monocrotalineinduced right ventricular hypertrophy in rats. Am J Physiol Heart Circ Physiol 2007;293:H1869-76.

52. Li S, Wang J, Xiao Y, et al. D-ribose: Potential clinical applications in congestive heart failure and diabetes, and its complications (Review). Exp Ther Med 2021;21:496.

53. Shecterle LM, Terry KR, St Cyr JA. Potential Clinical 
Benefits of D-ribose in Ischemic Cardiovascular Disease. Cureus 2018;10:e2291.

54. Cicero AFG, Colletti A. Nutraceuticals and Dietary Supplements to Improve Quality of Life and Outcomes in Heart Failure Patients. Curr Pharm Des 2017;23:1265-72.

55. Wagner S, Herrick J, Shecterle LM, et al. D-ribose, a metabolic substrate for congestive heart failure. Prog Cardiovasc Nurs 2009;24:59-60.

56. Herrick J, St Cyr J. Ribose in the heart. J Diet Suppl 2008;5:213-7.

57. Ingwall JS, Weiss RG. Is the failing heart energy starved?

Cite this article as: Krueger KJ, Rahman FK, Shen Q, Vacek J, Hiebert JB, Pierce JD. Mitochondrial bioenergetics and D-ribose in HFpEF: a brief narrative review. Ann Transl Med 2021;9(19):1504. doi: 10.21037/atm-21-2291
On using chemical energy to support cardiac function. Circ Res 2004;95:135-45.

58. Rooyackers OE, Adey DB, Ades PA, et al. Effect of age on in vivo rates of mitochondrial protein synthesis in human skeletal muscle. Proc Natl Acad Sci U S A 1996;93:15364-9.

59. Chan XC, Black CM, Lin AJ, et al. Mitochondrial protein turnover: methods to measure turnover rates on a large scale. J Mol Cell Cardiol 2015;78:54-61.

60. Chen X, Su T, Chen Y, et al. d-Ribose as a Contributor to Glycated Haemoglobin. EBioMedicine 2017;25:143-53. 